\title{
Preparation of the International Standardization on MOTIVATIVE Exercise Enforcement
}

\author{
Yoshiko Morita, MD \\ Director of the Department of the Rehabilitation Medicine, \\ Okayama rehabilitation hospital
}

I have worked as a specialist of Rehabilitation Medicine since graduation of Okayama University medical course. So I've been working for 45 years long in clinical fields. Many environments have been changed almost in this half century.

The biggest change is the shape of distribution map of population. It means that the lesser young aged versus the more senior aged people have made both the social economy and system unstable. The reason of instability is that social workers continue to decrease definitely from now on in this century long. The recipient will exceed the provider of medical and care insurances.

In Japanese honorable health care system covering whole nation, we worry about the medical and welfare funds exhausted near future. We can't rely on the public medical or care insurance and put ourselves at ease within our days. We baby boomers must decide to stop our children assume too much burden to take care of us. We must decide to devise to delay inactivity by ourselves reasonably. The expensive tools must not save all the people rather than the convenient ones. The devices we use in Motivative Exercise just answer these conditions. Many senior frail persons in a local region will be able to continue Motivative Exercise to keep the independent life anyway.

We succeeded to have chances to certify activated brain while exercising Motivative Exercise,by fNIRS and fMRI. The result from analyzing data getting by these examinations were not so easy to explain. But I strongly declare the group exercise will adapt much better than elderly health training better than personal training.

We provide our coming elderly to keep our ADL well by ourselves using Motivative Exercise. 